\title{
Médiévales
}

Langues, Textes, Histoire

74 | printemps 2018

Chanter la Croisade albigeoise

\section{Damien COULON et Christine GADRAT-OUERFELLI (dir.), Le Voyage au Moyen Âge. Description du monde et quête individuelle}

Aix-en-Provence, Presses universitaires de Provence (« Le temps de l'histoire »), 2017, 180 p.

Michèle Guéret-Laferté

\section{(2) OpenEdition}

Journals

Édition électronique

URL : https://journals.openedition.org/medievales/8872

DOI : $10.4000 /$ medievales.8872

ISSN : $1777-5892$

Éditeur

Presses universitaires de Vincennes

Édition imprimée

Date de publication : 15 juillet 2018

Pagination : 196-198

ISBN : 978-2-84292-837-7

ISSN : 0751-2708

Référence électronique

Michèle Guéret-Laferté, « Damien coulon et Christine gadrat-ouerfelli (dir.), Le Voyage au Moyen Âge. Description du monde et quête individuelle », Médiévales [En ligne], 74 I printemps 2018, mis en ligne le 10 août 2018, consulté le 24 avril 2022. URL : http://journals.openedition.org/medievales/8872 ; DOI https://doi.org/10.4000/medievales.8872

Ce document a été généré automatiquement le 24 avril 2022.

Tous droits réservés 


\section{Damien COULON et Christine GADRAT- OUERFELLI (dir.), Le Voyage au Moyen Âge. Description du monde et quête individuelle}

Aix-en-Provence, Presses universitaires de Provence (« Le temps de l'histoire »), 2017, $180 \mathrm{p}$.

Michèle Guéret-Laferté

\section{RÉFÉRENCE}

Damien Coulon et Christine GADRAT-OUERFELli (dir.), Le Voyage au Moyen Âge. Description du monde et quête individuelle, Aix-en-Provence, Presses universitaires de Provence (« Le temps de l'histoire »), 2017, $180 \mathrm{p}$.

1 Le volume, qui réunit les contributions présentées lors de deux journées d'étude à Strasbourg et à Aix-en-Provence en janvier et en mai 2014, explore la très grande diversité des récits de voyage médiévaux sous deux aspects habituellement opposés et pourtant solidaires, leur apport à la connaissance géographique du monde et leur composante autobiographique. En effet, par sa confrontation à l'ailleurs et à l'étranger, le voyageur est souvent amené à un questionnement sur sa propre identité. Sans doute les récentes recherches menées sur le processus d'individuation, tel qu'il s'effectue notamment entre le $\mathrm{XIII}^{\mathrm{e}}$ et le $\mathrm{Xv}^{\mathrm{e}}$ siècle, soulignent-elles les multiples nuances qui caractérisent cette évolution, comme le confirment d'ailleurs les textes de voyage pris en compte dans ce volume.

2 La première section privilégie le rapport entre récit de voyage et savoir géographique. Julia Roumier a étudié le Libro ultramarino, compilation de la fin du XIV siècle qui réunit des traductions castillanes de l'Historia orientalis de Jacques de Vitry, de la Lettre du Prêtre Jean et de la relation d'odoric de Pordenone. Les textes ont été remaniés de façon 
à cultiver la curiosité des lecteurs en insistant sur les merveilles, qu'elles concernent les populations étrangères ou les marchandises de prix. La géographie s'écrit ici davantage dans la perspective de divertir. En revanche, la traduction latine vulgate du Voyage de Mandeville, qui connaît une vaste diffusion européenne (trente-neuf manuscrits et plusieurs éditions au $\mathrm{xv}^{\mathrm{e}}$ siècle), met en évidence, selon Marianne o'Doherty, un ordre géographique qui structure le monde : le remanieur n'hésite pas à modifier la présentation des lieux et des itinéraires afin d'en accroître la cohérence. L'étude des gloses de quelques manuscrits de cette version confirme l'intérêt de leurs lecteurs pour la géographie puisque, dans leurs commentaires marginaux, ils s'efforcent de relier les informations données par Mandeville à celles que transmettent d'autres textes géographiques reconnus pour leur fiabilité. Mais, comme le montre Nathalie Bouloux dans son étude du statut du récit de voyage dans la géographie humaniste du $\mathrm{Xv}^{\mathrm{e}}$ siècle, la difficulté d'intégrer les connaissances nouvelles à la culture savante livresque, notamment à celle qui nous a été léguée par les auteurs antiques, peut poser problème. Si, d'un côté, les progrès de la cartographie, notamment pour ce qui concerne l'Asie, sont indéniablement liés à l'utilisation des relations de Marco Polo et de Niccolò de' Conti, on constate de l'autre côté que Poggio Bracciolini, grâce auquel nous avons pourtant conservé le compte rendu de voyage de Niccolò de' Conti dans le livre IV de son De varietate fortunae, et Enea Silvio Piccolomini, dans son De Asia, ne font aucune référence à Marco Polo. L'expérience du voyage ne peut se dire et s'écrire que dans le cadre et dans les termes d'une tradition propre à la culture du voyageur ou de celui qui décide de la transmettre. C'est bien ce qu'illustrent les deux premières communications ouvrant la deuxième section du volume, "Voyage et expérience du monde », qui traitent de textes concernant des aires culturelles diverses, byzantine pour la première, arabo-musulmane pour la seconde. Élisabeth Malamut, qui examine le récit du voyage en Serbie de Nicéphore Grégoras, accompli en 1327 dans le cadre d'une ambassade envoyée par Andronic II, remarque que l'auteur truffe son texte de références littéraires (à Homère, entre autres) et scripturaires, et que l'altérité des populations rencontrées est fortement soulignée. Quant à Yann Déjugnat, qui retrace l'évolution de la rihla entre le $\mathrm{xII}^{\mathrm{e}}$ et le $\mathrm{xIV}^{\mathrm{e}}$ siècle, il montre que cette forme littéraire entendait d'abord présenter le voyage comme une quête du savoir tout en magnifiant l'unité de l'empire islamique, en identifiant son centre et en explorant ses confins. Cette géographie spirituelle se double d'une observation du monde qui met en valeur les merveilles, comme chez al-Gharnāțī, ou des espaces nouveaux, comme chez Ibn Batțūta, ou donne lieu à une réflexion sur la civilisation comme chez Ibn Khaldūn, qui oppose sédentaires et bédouins. L'histoire de la rihla ne serait donc pas celle d'une divergence entre voyage et géographie, comme on avait pu le penser, mais plutôt celle d'une convergence, même si la composante idéologique et religieuse demeure importante dans cette littérature. Retrouvant la culture latino-européenne, Christine Gadrat-Ouerfelli prend pour objet le récit de pèlerinage de Ludolf de Sudheim qui a connu une diffusion importante, bien que l'identité de l'auteur soit difficile à cerner. Il rend compte de son voyage accompli entre 1336 et 1341 dans un texte qui témoigne de sa curiosité : il n'hésite pas à compléter ses informations en recourant aux ouvrages de son compatriote Guillaume de Boldensele et de l'Arménien Hayton et à évoquer des lieux qu'il n'a pas visités mais dont il a entendu parler. C'est aussi un récit de pèlerinage que nous livre Bernhard von Breydenbach, étudié par Emmanuelle Vagnon, mais ici le voyage à Jérusalem, entrepris entre 1483 et 1484, est destiné d'emblée à faire l'objet d'un livre, qui plus est illustré, puisque le pèlerin se fait accompagner par un 
peintre et graveur, Erhard Reuwich. En fait, comme on le voit avec le récit bien différent rapporté par Félix Fabri, qui était leur compagnon de route, les pèlerins s'approprient le même parcours (d'autant plus réglé que la visite des Lieux saints a été codifiée par les franciscains au milieu du xIV siècle et qu'elle est organisée par leurs soins) afin de produire le récit d'une expérience singulière. Emmanuelle Vagnon s'attache à montrer comment l'œuvre de Breydenbach propose une recomposition de l'imaginaire géographique du pèlerinage, notamment grâce aux vues paysagères de Reuwich qui produisent un " effet de réel» par la manipulation visuelle de la perspective. La dernière section de l'ouvrage, intitulée "Voyage et processus d'individuation ", s'interroge sur la composante autobiographique de quelques récits de voyage. Benoît Tock étudie la Translatio sanctae Monicae de Gautier d'Arrouaise. Ce chanoine du diocèse d'Arras, envoyé en 1161 en mission par son abbé auprès du pape Alexandre III, en aurait profité pour rapporter d'Ostie les reliques de la mère de saint Augustin. Si le voyageur est très présent dans le récit de son aventure, c'est avant tout pour garantir l'authenticité du produit de son larcin, et peut-être aussi sous l'influence des Confessions de saint Augustin qu'il connaît bien. Le récit de pèlerinage étudié par Damien Coulon a une destination plus originale puisqu'il s'agit du Purgatoire de saint Patrick, localisé en Irlande. Son auteur, le vicomte Ramon de Perellòs, qui écrit vers 1398, associe à son récit de voyage une évocation de sa rencontre au Purgatoire avec le roi de Catalogne Jean ${ }^{\text {er }}$, décédé brutalement en 1396, ainsi qu'une traduction du Tractatus de Purgatorio sancti Patricii de Henry de Saltrey (vers 1180). Dans cet ouvrage devenu un classique de la littérature catalane et qui s'apparente aux Voyages dans l'Audelà, Damien Coulon interroge la composante autobiographique : l'auteur, qui a servi Jean I ${ }^{\mathrm{er}}$ après avoir servi son père Pierre IV, écrit avant tout pour la cour aragonaise, d'abord pour se disculper de certaines accusations portées contre lui, et puis pour s'offrir comme médiateur entre le roi défunt et ses anciens sujets ; c'est aussi la mémoire de la classe chevaleresque que, ce faisant, il célèbre. La dernière contribution du volume, due à Béatrice Dansette, met en évidence ce même trait dans le récit que nous a laissé Nompar de Caumont de son pèlerinage à Jérusalem en 1419-1420. Le prologue, de même que les remarques personnelles ajoutées au récit de la visite très balisée des Lieux saints, concernant son adoubement au saint Sépulcre et la création de l'ordre de l'Écharpe d'azur, montrent comment le voyage a contribué à fortifier ses sentiments religieux, mais surtout à affirmer son sentiment d'appartenance à la classe chevaleresque en ces années dramatiques pour la noblesse après le désastre d'Azincourt et en pleine guerre de Cent Ans.

3 Ce volume a donc le mérite de présenter une grande variété de relations de voyage médiévaux, certains bien connus, d'autres beaucoup moins, et d'illustrer la plasticité de cette forme littéraire. Comme l'affirme Patrick Gautier Dalché dans ses Conclusions, " les récits de voyage sont des textes et doivent être étudiés en tant que tels ». En effet, leur structuration narrative obéit à des impératifs très variables, d'où la nécessité d'en éclairer scrupuleusement le contexte afin de les interpréter au mieux. 


\section{AUTEURS}

MICHĖLE GUÉRET-LAFERTÉ

Université de Rouen-Normandie 